\title{
ON THE GROUP GENERATED BY A FREE SEMIGROUP
}

\author{
K. I. APPEL AND F. M. DJORUP
}

The following theorem answers a question proposed by E. Granirer $^{1}$ and A. H. Frey.

Theorem. A group generated by a two generator free semigroup need not be free.

Let $H$ be the free group on generators $a$ and $b, K$ the free semigroup on $a$ and $b$ and $N$ the normal subgroup of $H$ generated by $a b^{-1} a b^{-1}$. Let $G=H / N$. In particular, we will show that $K$ is embedded in $G$ by the natural embedding.

We will use $c$ for $a, b, a^{-1}$, or $b^{-1}$. When $c$ and $d$ are used in the same word, $(c, d)$ will represent $(a, b),(b, a),\left(a^{-1}, b^{-1}\right)$ or $\left(b^{-1}, a^{-1}\right)$. If $W$ is any word on $a, b, a^{-1}, b^{-1}$ we say that $W$ has $n$ changes of sign if $W=P_{1} P_{2}^{-1} \cdots P_{n+1}^{(-1)^{n}}$ or $P_{1}^{-1} P_{2} \cdots P_{n+1}^{(-1)^{n+1}}$, where each $P_{i}$ is a nonempty word on $a$ and $b$.

We remark that if the semigroup generated by $a$ and $b$ were not free in $G$ then there would be two distinct words $P_{1}$ and $P_{2}$ on $a$ and $b$ representing the same member of $G$. Hence there would be a nontrivial word $P_{1} P_{2}^{-1}$ which would represent an element of $N$ and have precisely one change of sign. The construction which follows implies that every representative of a nontrivial element of $N$ has at least three changes of sign.

Let $U$ be the collection of all sets $B$ of words on $a, a^{-1}, b, b^{-1}$ obeying the following rules:

1. $B$ contains $e$ (the empty word), $a a^{-1}, b b^{-1}, b^{-1} b, a^{-1} a$ (the inverse pairs) and $a^{-1} b a^{-1} b, a b^{-1} a b^{-1}, b^{-1} a b^{-1} a, b a^{-1} b a^{-1}$ (which we shall call the basic words).

2. If $V_{1}$ and $V_{2} V_{3}$ are members of $B$ and $V_{1}$ and $V_{2}$ are nonempty, then $V_{2} V_{1} V_{3} \in B$ (N.B. $V_{2}$ and $V_{3}$ need not be members of $B$ ).

Let $A=\cap U$ (i.e., the smallest set satisfying rules 1 and 2). Clearly $A \in U$ and every element of $A$ can be constructed from the elements given in rule 1 by a finite number of applications of rule 2 .

Lemma 1. A contains all products of formal conjugates of the basic words.

Received by the editors March 2, 1963.

1 Proposed in the University of Illinois problem book. Originally proposed in the doctoral dissertation of A. H. Frey. 
Proof. Rule 2 applied to inverse pairs $V_{2} V_{3}$ permits conjugation of $V_{1}$. Rule 2 applied with $V_{3}$ empty permits multiplication.

Corollary. Each element of $N$ is represented by an element of $A$.

Definition. A word $V$ on $a, a^{-1}, b, b^{-1}$ is reduced unless $V$ is of the form $S_{c c}^{-1} T$.

Lemmas 2 through 4 will show that if $W$ is an element of $A$ and $V$ is obtained from $W$ by successive deletions of inverse pairs, $V$ is an element of $A$ and furthermore $V$ is either empty or has at least three changes of sign. Trivially, we can consider the word $P_{1} P_{2}^{-1}$ of paragraph 3 to be reduced, hence not in $A$. But, in view of Lemma 1, the unique reduced word representing a member of $N$ would be in $A$. This will prove the theorem.

We will prove Lemmas 2, 3 and 4 by strong induction on the length of the word. In each case the basis of the induction is trivial, and we will assume that the conclusion holds for words shorter than the word under consideration. We adopt the following convention: An equation of the form $V=V_{2} V_{1} V_{3}$ means $V \in A, V_{2} V_{3} \in A, V_{1} \in A, V_{1}$ and $V_{2}$ are nonempty.

LEMMA 2. Every nonempty reduced word in $A$ has at least three changes of sign.

Proof. Let $V$ be a nonempty reduced word in $A$. If $V$ is a basic word, $V$ has 3 changes of sign. If $V$ is reduced and not basic, then $V=V_{2} V_{1} V_{3}$. Therefore, $V_{1}$ is reduced and has, by the induction hypothesis, three changes of sign. Hence $V$ does also.

Lemma 3. $c V \in A$ if and only if $V c \in A$.

Proof. If $c V \in A$ and is an inverse pair or basic word, $V c \in A$. If $c V=V_{2} V_{1} V_{3}=c U V_{1} V_{3}$ then $c U V_{3} \in A$ by our convention, $U V_{3} c \in A$ by inductive hypothesis on shorter words and $U V_{1} V_{3} c=V c \in A$ by rule 2. The proof of the other implication is similar.

Corollary. If $V_{1} V_{2} \in A$ then $V_{2} V_{1} \in A$.

In proving the next lemma, we will use the following notation: $P * Q-2 \rightarrow R$ means that $R \in A$ by an application of rule 2 to $P$ and $Q$ which are elements of $A . P-i \rightarrow R$ means that $P$ is a word in $A$ which is shorter than the word being considered in the inductive step and the inductive hypothesis may be applied to show that $R \in A$. $P-r \rightarrow R$ means that $P \in A$ and $P=V_{2} V_{1} V_{3}$ as in rule 2, with $R=V_{2} V_{3}$, whence $R \in A$.

Lemma 4. If $V=c c^{-1} S \in A$ then $S \in A$. 
Proof. If $V$ is an inverse pair, $S$ is empty and in $A . V$ cannot be a basic word. Hence we can assume $V=V_{2} V_{1} V_{3}$.

Case 1. $V_{2}=c c^{-1} T$ so $V_{2} V_{1} V_{3}=c c^{-1} T V_{1} V_{3}-r \rightarrow c c^{-1} T V_{3}-i \rightarrow T V_{3}$ and $T V_{3} * V_{1}-2 \rightarrow T V_{1} V_{3}=S$.

Case 2. $V_{2}=c, V_{2} V_{1} V_{3}=c c^{-1} R V_{3}$.

Subcase 1. $V_{1}$ and $V_{2} V_{3}$ both arise from rule 1 .

a. Both are inverse pairs so $V=c c^{-1} c c^{-1}, S=c c^{-1} \in A$.

b. $V_{1}$ is an inverse pair, $V_{2} V_{3}$ a basic word, $V=c c^{-1} c d^{-1} c d^{-1}$, $S=c d^{-1} c d^{-1} \in A$.

c. $V_{2} V_{3}$ is an inverse pair, $V_{1}$ is a basic word, $V=c c^{-1} d c^{-1} d c^{-1}$, $S=d c^{-1} d c^{-1} \in A$.

d. $V_{2} V_{3}$ and $V_{1}$ are basic words, $V=c c^{-1} d c^{-1} d d^{-1} c d^{-1}$. $S=d c^{-1} d d^{-1} c d^{-1}$ arises from two applications of rule 2 to inverse pairs and hence is in $A$.

Subcase 2. $V_{1}=U_{2} U_{1} U_{3}$, so $U_{2}=c^{-1} T$ and $V_{2} V_{1} V_{3}=c c^{-1} T U_{1} U_{3} V_{3}$ $-r \rightarrow c V_{3}$. But $U_{2} U_{1} U_{3}=c^{-1} T U_{1} U_{3}-r \rightarrow c^{-1} T U_{3}$ and $c^{-1} T U_{3} * c V_{3}$ $-2 \rightarrow c c^{-1} T U_{3} V_{3}-i \rightarrow T U_{3} V_{3}$. Hence $T U_{3} V_{3} * U_{1}-2 \rightarrow T U_{1} U_{3} V_{3}=S$.

Subcase 3. $V_{2} V_{3}=U_{2} U_{1} U_{3}$, so $U_{2}=c T$ and $U_{2} U_{1} U_{3}=c T U_{1} U_{3}$ $-r \rightarrow c T U_{3}$ and $c T U_{3} * c^{-1} R-2 \rightarrow c c^{-1} R T U_{3}-i \rightarrow R T U_{3}$. Hence $R T U_{3}$ * $U_{1}-2 \rightarrow R T U_{1} U_{3}=S$.

Corollary. If $V_{1} c c^{-1} V_{2} \in A$, then $V_{1} V_{2} \in A$.

Proof. Use corollary to Lemma 3.

This corollary asserts that reduction is permitted in $A$ and the theorem is proved.

UNIVERSITY OF ILLINOIS 